\title{
Identification of novel biomarker as citrullinated inter-alpha-trypsin inhibitor heavy chain 4, specifically increased in sera with experimental and rheumatoid arthritis
}

Hoshimi Kawaguchi ${ }^{1}$, Isao Matsumoto ${ }^{1 *}$, Atsumu Osada ${ }^{1}$, Izumi Kurata ${ }^{1}$, Hiroshi Ebe ${ }^{1}$, Yuki Tanaka', Asuka Inoue ${ }^{1}$ Naoto Umeda ${ }^{1}$, Yuya Kondo ${ }^{1}$, Hiroto Tsuboi ${ }^{1}$, Yasuhiro Shinkai ${ }^{2}$, Yoshito Kumagai ${ }^{2}$, Akihito Ishigami ${ }^{3}$ and Takayuki Sumida'

\begin{abstract}
Background: Anticitrullinated protein antibodies (ACPA) and citrullinated proteins play key roles in the pathogenesis of rheumatoid arthritis (RA). Many candidate citrullinated antigens have been identified in joints, but citrullinated proteins in sera are mostly uncertain in patients with RA. We explored the expression of citrullinated proteins in joints and sera of experimental arthritis, and we further investigated their specific expression correlated with the disease activity in patients with RA.
\end{abstract}

Methods: Citrullinated protein expression in tissues was examined by IHC in peptide glucose-6-phosphate isomerase-induced arthritis ( $p G \mid A)$. Serum citrullinated proteins from pGIA were examined by Western blotting, and the sequence was identified by MS. With the same methods, serum citrullinated proteins were analyzed in patients with RA, primary Sjögren's syndrome, systemic lupus erythematosus, and osteoarthritis as well as in healthy subjects, by Western blotting and MS. In patients with RA, the relationship between the expression of the identified protein (inter-alpha-trypsin inhibitor heavy chain 4 [ITIH4]) and clinical features was evaluated, and the levels of citrullinated ITIH4 were compared before and after biological treatment. The antibody response against citrullinated ITIH4 peptide was measured by enzyme-linked immunosorbent assay.

Results: Citrullinated proteins were detected specifically in arthritic joints and sera from PGIA relative to controls. In sera, a common band of citrullinated protein at $120 \mathrm{kDa}$ was revealed, and it fluctuated in parallel with arthritis score of pGIA by Western blotting. Interestingly, in $82 \%$ of RA patient sera, similar bands of citrullinated protein were specifically detected. These proteins were identified as citrullinated ITIH4, and especially the R438 site was commonly citrullinated between mice and humans. Citrullinated ITIH4 levels were associated with clinical parameters such as C-reactive protein (CRP), rheumatoid factor, and Disease Activity Score in 28 joints as measured by CRP in patients with RA. Its levels were decreased in correlation with the reduction of disease activity score after effective treatment in patients with RA. Moreover, antibody response to citrullinated epitope in ITIH4 was specifically observed in patients with RA.

Conclusions: Our results suggest that serum citrullinated ITIH4 was specifically increased in patients with RA and could be a novel biomarker for assessing disease activity in patients with RA.

Keywords: Rheumatoid arthritis, Citrullinated proteins, Inter-alpha-trypsin inhibitor heavy chain 4, PAD

\footnotetext{
* Correspondence: ismatsu@md.tsukuba.ac.jp

'Department of Internal Medicine, Faculty of Medicine, University of Tsukuba,

1-1-1 Tennodai, Tsukuba 305-8575, Japan

Full list of author information is available at the end of the article
} 


\section{Background}

Rheumatoid arthritis (RA) is an autoimmune disease characterized by the inflammation and destruction of joints and surrounding tissues [1]. Most patients with RA are positive for anticitrullinated protein antibodies (ACPA), and these antibodies are highly specific for RA diagnosis [2]. ACPA appear years before the onset of clinical RA and are the predictive factor of joint radiographic progression [2]. In the mouse model, it was reported that ACPA appeared prior to the development of clinical disease in collagen-induced arthritis, and administration of anticitrullinated fibrinogen antibodies enhanced arthritis [3]. Thus, ACPA is considered to be involved in the pathogenesis of RA.

ACPA recognize various citrullinated proteins, such as vimentin, $\alpha$-enolase, fibrinogen, fibronectin, and glucose6 -phosphate isomerase (GPI) [2, 4]. High levels of citrullinated proteins have been found in RA joints $[5,6]$. Also, citrulline-specific $\mathrm{T}$ cells were increased in peripheral blood of patients with RA and decreased by therapy [7]. In murine models, immunization of citrullinated fibrinogen induced arthritis in HLA-DRB1*0401-transgenic mice, but unmodified fibrinogen did not [8]. However, we do not know what citrullinated antigens specifically revealed in joints and sera in arthritis.

GPI is a ubiquitous glycolytic enzyme and was identified as a pathogenic autoantigen in $\mathrm{K} / \mathrm{BxN}$ arthritic mice [9]. Immunization with recombinant human GPI could induce arthritis in DBA/1 mice [10]. A major epitope of GPI-specific $\mathrm{CD}_{4}^{+} \mathrm{T}$ cells in glucose-6-phosphate isomerase-induced arthritis (GIA) was identified as peptide 325-339 glucose-6-phosphate isomerase (pGPI), and immunization with pGPI can also induce arthritis just as GIA does in DBA/1 mice (peptide 325-339 glucose-6phosphate isomerase-induced arthritis [pGIA]) [11]. CD4 ${ }^{+}$ T cells play a critical role in GIA $[10,12]$, and the effect of biological agents in GIA is similar to RA [12, 13]. However, the involvement of ACPA and citrullinated proteins have not investigated in this model.

In the present study, we explored the expression and commonality of citrullinated proteins in pGIA and patients with RA, and we further investigated its correlation with RA disease activity. We show the production of ACPA, and we detected citrullinated proteins in arthritic joints and sera in pGIA. Serum citrullinated protein in pGIA was identified as citrullinated inter-alphatrypsin inhibitor heavy chain 4 (ITIH4). The expression of ITIH4 was increased in inflamed synovium at day 14 in pGIA, suggesting the association with arthritis. In addition, bands of the same size were also detected in sera of patients with RA by Western blotting, which were confirmed as citrullinated ITIH4 as well. The levels of citrullinated ITIH4 were specifically increased in sera of patients with RA and were clearly associated with RA disease activity. Moreover, antibody response to citrullinated epitope in ITIH4 was observed in patients with RA. Citrullinated ITIH4 could be a novel specific biomarker representing the disease activity of patients with RA.

\section{Methods \\ Mice}

Male DBA/1 mice were purchased from Charles River Japan (Tokyo, Japan), and used at 6-10 weeks of age. All mice were maintained under specific pathogen-free conditions at the University of Tsukuba. All experimental protocols were approved by the Institutional Animal Care and Use Committee of the University of Tsukuba, and all animal experiments were conducted in accordance with institutional ethics guidelines. All surgeries were performed under isoflurane anesthesia, and utmost care was taken to minimize suffering.

\section{Serum samples}

Serum samples were collected from Japanese patients with RA ( $n=60$, mean age 52.2 years, range 20-73 years, females $80 \%$ ) diagnosed by rheumatologists according to the 1987 American College of Rheumatology (ACR) classification criteria [14] or the 2010 ACR/European League Against Rheumatism classification criteria [15]. Serum samples of the disease control subjects were collected from Japanese patients with primary Sjögren's syndrome (SS) $(n=27$, mean age 58.8 years, range $26-80$ years, females 96\%), systemic lupus erythematosus (SLE) $(n=15$, mean age 33.9 years, range $16-55$ years, females $80 \%)$, or osteoarthritis (OA) $(n=12$, mean age 56.3 years, range $37-80$ years, females $67 \%)$. All the patients with SS were diagnosed by rheumatologists according to the 1999 Japanese Ministry of Health criteria for diagnosis of SS [16]. All the patients with SLE fulfilled the 1997 ACR classification criteria [17]. None of the patients with SS or SLE had overlapping RA. Serum samples were collected from healthy subjects (HS) $(n=30$, mean age 49.0 years, range $34-65$ years, females $80 \%$ ). Serum samples were also collected from 17 patients with RA before and 24 weeks after treatment with biologic drugs (infliximab, $n=9$; abatacept, $n=8$ ). All samples were collected at the University of Tsukuba Hospital after informed consent was obtained. This study was reviewed and approved by the ethics committee of the University of Tsukuba.

\section{Peptide GPI-induced arthritis}

DBA/1 mice were immunized with $25 \mu \mathrm{g}$ of pGPI (Invitrogen/Thermo Fisher Scientific, Carlsbad, CA, USA) in complete Freund's adjuvant (CFA) (BD Biosciences, San Jose, CA, USA). pGPI was emulsified with CFA at a 1:1 ratio (vol/vol), or PBS + CFA was prepared as a vehicle control. For induction of arthritis, $150 \mu$ l of the emulsion 
was injected intradermally at the base of the tails of the mice. Each mouse was also given an injection of $200 \mathrm{ng}$ of pertussis toxin (Sigma-Aldrich, St. Louis, MO, USA) intraperitoneally on days 0 and 2 after immunization to induce arthritis. Arthritis was assessed every other day and evaluated using a scale of $0-3$ for swelling and redness of each paw. The clinical score was the sum of the scores for four paws, as described previously [11].

\section{Measurement of anti-CCP antibodies in pGIA}

Sera were obtained on days 0-28 every week from mice immunized with pGPI or control, and antibodies were measured by enzyme-linked immunosorbent assay (ELISA). Sera were diluted 1:25 in dilution buffer and added to the 96-well plate (Immunoscan CCPlus test kit; Euro Diagnostica, Malmö, Sweden) for $1 \mathrm{~h}$ at room temperature. After a washing step, horseradish peroxidase (HRP)-conjugated polyclonal rabbit antimouse immunoglobulin (Dako, Carpinteria, CA, USA) diluted 1: 1000 was added for 30 minutes at room temperature. After another washing step, color was developed with 3,3',5,5' -tetramethylbenzidine (TMB) microwell peroxidase substrate (KPL/SeraCare, Milford, MA, USA). The optical density (OD) was measured at $450 \mathrm{~nm}$ by using a microplate reader.

A standard pool was obtained by mixing sera obtained from several mice on day 28 . The concentrations of antibodies in this pool were considered $100 \mathrm{U} / \mathrm{ml}$. A standard curve was obtained using serum dilutions, and the Michaelis-Menten equation was used to convert OD values into units, as described previously [18].

Measurement of anti-ITIH4 antibodies in patients with RA Native peptide ITIH $4_{428-447}$ and R438 citrullinated peptide ITIH4 4 428-447 were synthesized (serum, purity 95\%) and used in ELISA. Ninety-six-well plates (Nunc MaxiSorp; Thermo Fisher Scientific) were coated with $10 \mu \mathrm{g} / \mathrm{ml} \mathrm{pep-}$ tides for $12 \mathrm{~h}$ at $4{ }^{\circ} \mathrm{C}$. After washing and blocking steps, sera from patients with RA $(n=60)$ and HS $(n=30)$ were diluted 1:200 in 1\% bovine serum albumin (BSA) in PBS, then added for $2 \mathrm{~h}$ at room temperature. After a washing step, HRP-conjugated goat antihuman immunoglobulin $\mathrm{G}$ (IgG) (heavy and light chains $[\mathrm{H}+\mathrm{L}]$ ) (Abcam, Cambridge, MA, USA) diluted 1:10,000 was added for $1 \mathrm{~h}$ at room temperature. After washing, color was developed with TMB microwell peroxidase substrate (KPL/SeraCare). The OD was measured at $450 \mathrm{~nm}$ by using a microplate reader. The cutoff value was determined as the mean +2 SD of HS.

\section{Histopathological analysis}

Ankle joint tissue samples were harvested at days 0 and 14 from mice immunized with pGPI or control. They were fixed in neutralized 10\% formalin, embedded in paraffin, and sectioned. To detect citrullinated proteins, we prepared modification buffer by mixing Reagent A (20\% $\mathrm{H}_{2} \mathrm{SO}_{4}, 25 \% \mathrm{H}_{3} \mathrm{PO}_{4}$, and $0.025 \% \mathrm{FeCl}_{3}$ ) and Reagent $\mathrm{B}(1 \%$ diacetyl monoxime, $0.5 \%$ antipyrine, $1 \mathrm{M}$ acetic acid) at a 2:1 ratio ( $\mathrm{vol} / \mathrm{vol})$. The sections were covered with the modification buffer and incubated in a light-proof container at $37{ }^{\circ} \mathrm{C}$ for $2.5 \mathrm{~h}$ to modify citrulline residues. Then, the sections were incubated overnight at room temperature with rabbit anti-modified citrulline (AMC) polyclonal antibodies diluted 1:3200 in $2 \%$ BSA in PBS to detect modified citrulline residues [19]. The sections were also incubated with HRPconjugated goat antirabbit IgG $(\mathrm{H}+\mathrm{L})$ (Bio-Rad Laboratories, Hercules, CA, USA) for 30 minutes at room temperature. The sections were also stained with 3,3' -diaminobenzidine (DAB) (Nichirei Biosciences, Tokyo, Japan) and hematoxylin. To detect ITIH4 or macrophages, IHC analysis of the joint or liver (as a positive control of ITIH4 staining) sections was performed as described above, using rabbit antihuman ITIH4 antibodies (Abcam) diluted 1:1000 and rat antimouse F4/80 antibodies (BioLegend, San Diego, CA, USA) diluted 1:200 as primary antibodies and using rabbit-specific IHC polymer detection kit HRP/DAB (Abcam) and HRP-conjugated polyclonal rabbit antirat immunoglobulin (Dako) diluted 1:200 as secondary antibodies, respectively.

\section{Real-time qPCR analysis}

We extracted total RNA from the ankle joints of pGIA or control mice using the ISOGEN (Wako Pure Chemical Industries, Tokyo, Japan) extraction method according to the instructions provided by the manufacturer. The extracted RNA was reverse-transcribed to complementary DNA with random primers. We performed real-time qPCR using a TaqMan gene expression assay (Applied Biosystems/Thermo Fisher Scientific, Foster City, CA, USA), and the Padi4 (Mm01341658_m1) and glyceraldehyde 3-phosphate dehydrogenase (GAPDH) (NM 002046) primers. Real-time qPCR was carried out using an ABI 7500 analyzer (Applied Biosystems). The expression of GAPDH was used as the control.

\section{Western blot analysis}

Serum samples were obtained at days $0,7,14$, and 28 from mice immunized with pGPI or control, as well as from patients with RA, SS, SLE, or OA and from HS. For analysis of citrullinated protein expression, serum samples were loaded into each well $(50 \mu \mathrm{g}$ for SDSPAGE or $100 \mu \mathrm{g}$ for two-dimensional PAGE [2DPAGE]), separated by SDS-PAGE or 2D-PAGE, and transferred to polyvinylidene difluoride membranes. The modification buffer was added to the blots before incubation in a light-proof container at $37{ }^{\circ} \mathrm{C}$ for $2.5 \mathrm{~h}$ to modify citrulline residues, as described above. Blots 
were washed with $0.05 \%$ Tween 20 in Tris-buffered saline (TBST) and blocked with 5\% milk in TBST, then incubated overnight at $4{ }^{\circ} \mathrm{C}$ with AMC antibodies diluted 1:3200 in 5\% milk in TBST [19]. After a washing step, the blots were incubated with secondary antibody HRP-conjugated goat antirabbit IgG $(\mathrm{H}+\mathrm{L})$ (Bio-Rad Laboratories) diluted 1:5000 in 5\% milk in TBST for 1 $\mathrm{h}$ at room temperature. Densitometric analysis was carried out using an ImageQuant LAS 4000 densitometer (GE Healthcare Life Sciences, Marlborough, MA, USA). The band intensity was determined with ImageQuant TL software (GE Healthcare Life Sciences) and normalized using the value of one sample (pGIA or RA) as 10 . The difference of citrullinated ITIH4 levels from baseline to 24 weeks after treatment was normalized using each value at baseline. For analysis of ITIH4 expression, serum samples were loaded $10 \mu \mathrm{g} /$ well and Western blot analysis was performed as described above, using rabbit antihuman ITIH4 antibodies (Abcam) diluted 1:3000.

\section{Measurement of citrulline content}

Sera were obtained at days $0,7,14$, and 28 from mice immunized with pGPI or control, and citrulline contents were measured by using the color development reagent (COLDER) assay, as described previously [20]. Sera were diluted 1:100 in COLDER buffer $(50 \mathrm{mM} \mathrm{NaCl}, 10 \mathrm{mM}$ $\mathrm{CaCl}_{2}, 2 \mathrm{mM}$ dithiothreitol, and $100 \mathrm{mM}$ Tris, $\mathrm{pH}$ 7.4), and $60 \mu \mathrm{l}$ of samples were added to the 96-well plates (Nunc MaxiSorp). Then, $200 \mu \mathrm{l}$ of COLDER were added to detect citrullinated proteins. The samples were incubated for 30 minutes at $95{ }^{\circ} \mathrm{C}$. The OD was measured at $570 \mathrm{~nm}$ by using a microplate reader and compared with a standard curve of known citrulline concentrations. Data were normalized relative to the protein concentration in each sample.

\section{LC-MS}

Sera from pGIA mice, control mice, patients with RA, and HS were separated by 2D-PAGE, and gels were stained with GelCode Blue Stain Reagent (Thermo Fisher Scientific) for Coomassie brilliant blue staining. Gel slices were incubated overnight with MS-grade modified trypsin $(6.7 \mathrm{ng} / \mu \mathrm{l}$; Promega, Madison, WI, USA) at $37{ }^{\circ} \mathrm{C}$. The resultant peptides were analyzed with the nanoACQUITY ultrahigh-performance LC (UPLC) system (Waters, Milford, MA, USA). Data were collected in centroid mode from mass-to-charge ratios $(\mathrm{m} / \mathrm{z}) 50$ to 1990 . All analyses were acquired with an independent reference. BiopharmLynx version 1.2 software (Waters) was used for baseline subtraction and smoothing, de-isotoping, de novo peptide sequence identification, and database searches.

\section{Statistical analysis}

All data were expressed as mean \pm SEM. Differences between groups were evaluated for statistical significance using Student's $t$ test. The Kruskal-Wallis test was used for evaluation of band intensity among the five subject groups. $P$ values less than 0.05 were considered significant. Statistical analyses were performed using IBM SPSS Statistics software (IBM, Armonk, NY, USA).

\section{Results \\ Overexpression of ACPA and citrullinated proteins in joints and sera in PGIA}

First, we investigated the production of ACPA in pGIA. $\mathrm{DBA} / 1$ mice were immunized with pGPI, and we induced symmetrical polyarthritis characterized by severe swelling of the limb joints (Additional file 1: Figure S1a). ACPA titers were measured in pGIA sera by ELISA, and they were significantly higher than those of control mice (Additional file 1: Figure S1b).

Then, we evaluated the expression of citrullinated proteins in pGIA because high titers of autoantibodies against citrullinated proteins were detected. Ankle joint sections were stained with AMC antibodies by IHC. Citrullinated proteins were detected in joints at day 14 (the peak arthritic phase) and expressed in areas of synovial hyperplasia in pGIA but not in controls (Fig. 1a). Citrullinated proteins were not expressed in several other tissues examined (e.g., lung, spleen, lymph node) (data not shown). We analyzed the gene expression of Padi4, which is an enzyme that catalyzes protein citrullination, in articular tissue samples from pGIA by qPCR. At day 14, Padi4 levels tended to be higher in the arthritic joints of pGIA mice than in control mice, albeit insignificantly (Additional file 2: Figure S2a).

In our previous study, PAD4 levels were increased in sera of patients with RA [21], so we next explored the expression of citrullinated proteins in sera. Western blot analysis of serum samples using AMC antibodies identified citrullinated proteins in pGIA mice but in none of the control mice (Fig. 1b). Interestingly, the band of citrullinated protein was detected as a common band at $\sim 120 \mathrm{kDa}$. The band was detected from the prearthritic phase and with significantly stronger intensity in the peak arthritic phase, then it decreased in line with the self-limited arthritis (Fig. 1c). Whereas citrullinated proteins were detected in serum samples (Additional file 3: Figure S3a, right), they were not detected when Western blot analysis was performed without modification of citrulline residues (Additional file 3: Figure S3a, left), suggesting that the detected band was really specific for citrulline.

To further confirm the serum levels of citrullinated proteins, we measured citrulline contents in pGIA sera using the COLDER assay. Serum citrulline content tended to be 

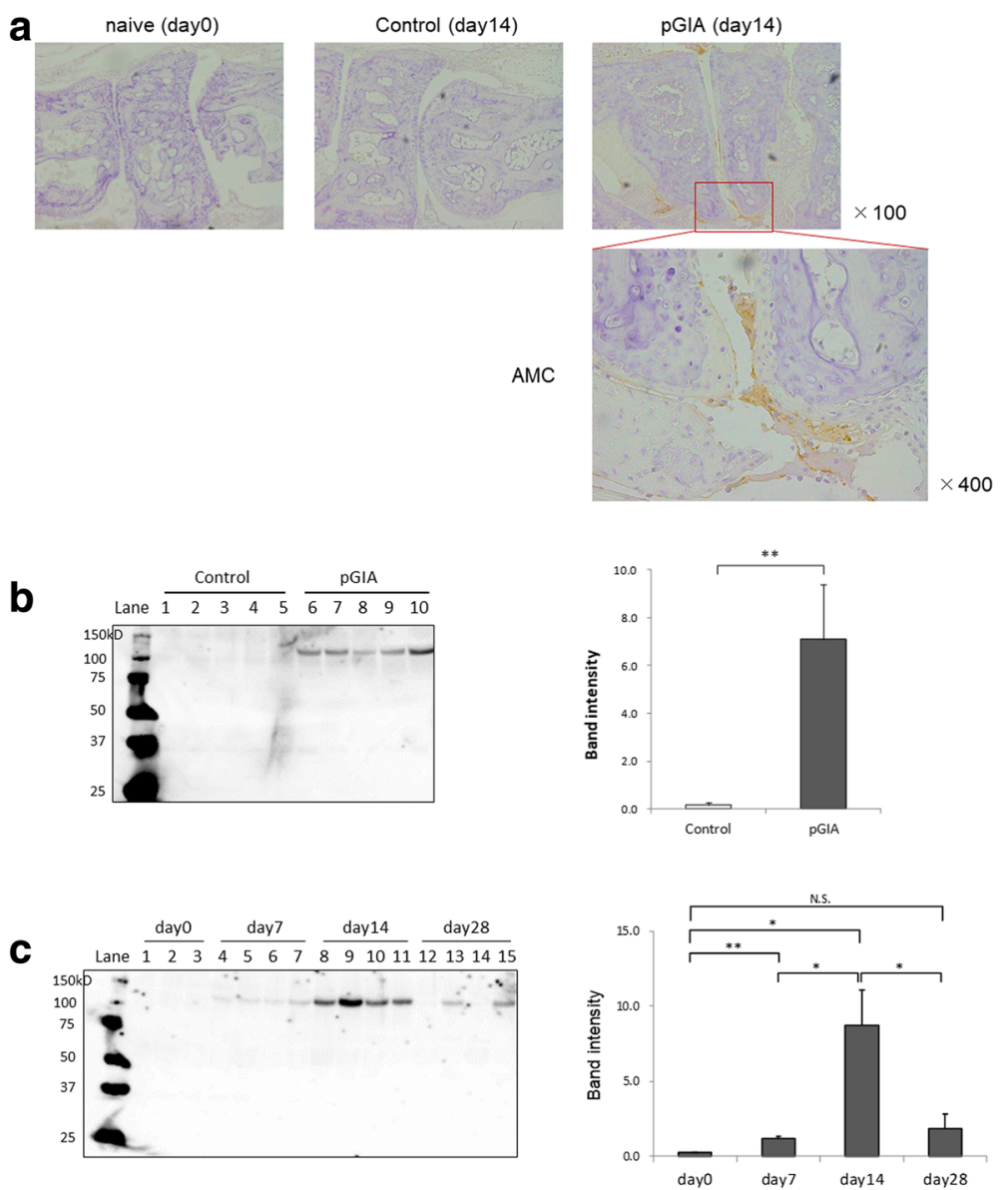

Fig. 1 The increase of citrullinated proteins in arthritic joints and sera in peptide glucose-6-phosphate isomerase-induced arthritis (pGIA). a Joint sections day 0 and day 14 were immunohistochemically stained with anti-modified citrulline (AMC) antibodies in pGIA or control mice. Citrullinated proteins were detected at day 14 in pGIA mice (indicated by boxed area) but not in control mice. The bottom right image is a magnified view of the boxed area in the joint image at day 14 of pGIA. Original magnification $\times 100$ and $\times 400$ as marked. The experiments were performed on at least three different samples with similar results. $\mathbf{b}$ Sera from $\mathrm{pGIA}$ and control mice at day 14 subjected to Western blot analysis using AMC antibodies. Citrullinated proteins were detected as a common band at $\sim 120 \mathrm{kDa}$ in sera from pGIA mice but not from control mice. Lanes 1-5: control mice; lanes 6-10: pGIA mice. Representative blots are shown at left; data at right are mean \pm SEM of band intensity $(n=9) .{ }^{* *} p<0.01$. c Sera from pGIA mice subjected to Western blot analysis. Citrullinated proteins appeared from day 7, increased at day 14, and decreased at day 28. Lanes 1-3: day 0; lanes 4-7: day 7, lanes 8-11: day 14, lanes 12-15: day 28. Representative blots are shown at left; data at right are mean \pm SEM of band intensity $(n=4)$. ${ }^{*} p<0.05,{ }^{* *} p<0.01$

higher in pGIA at day 14 than in the controls (Additional file 3: Figure S3b). These results suggest that citrullinated proteins appeared in joints and sera in association with arthritis in pGIA.

\section{Identification of citrullinated ITIH4 in sera and increase of ITIH4 in arthritic joints in pGIA}

To identify the $120 \mathrm{kDa}$ citrullinated protein in sera, we analyzed the protein by MS. First, we separated the serum samples of pGIA by 2D-PAGE and confirmed the spot of citrullinated proteins at $\sim 120 \mathrm{kDa}$ by Western blot analysis (Fig. 2a, right). Second, the spot in the gels was sliced after Coomassie brilliant blue staining (Fig. 2a, left), digested with trypsin, and analyzed by nanoUPLC-MS to identify the protein. ITIH4 was identified with high coverage (Table 1), and we examined citrulline modifications by nanoUPLC-MS ${ }^{\mathrm{E}}$. Citrullination of several sites containing R438 were identified in ITIH4 from pGIA (Table 1 and Fig. 2b). Analysis of the samples by MS after SDS-PAGE (Additional file 4: Figure S4a) also confirmed the presence of ITIH4 with high coverage, as well as citrullination at R438 in ITIH4 (Additional file 4: Figure S4b).

To further investigate the association between sera and arthritic joints, we explored the expression of ITIH4 in joints in pGIA. Ankle joint sections were stained with anti-ITIH4 antibodies by IHC, and liver sections were stained as positive controls to detect ITIH4 (Additional file 5: Figure S5a). In naïve mice, ITIH4 was not detected in joints, but ITIH4 was clearly expressed in inflamed synovium at day 14 in pGIA (Fig. 2c). Macrophages 
a

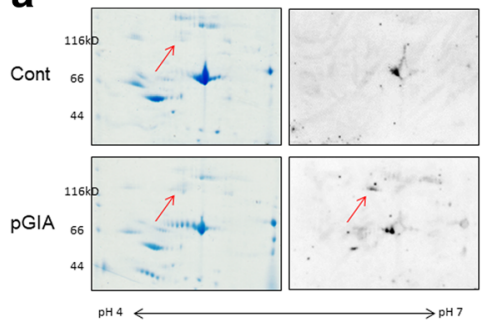

C
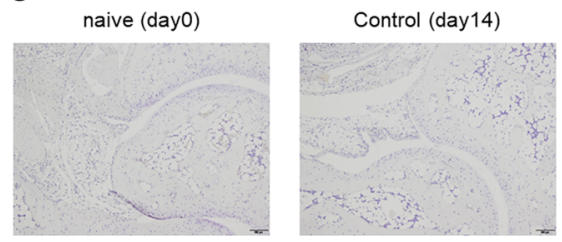

d
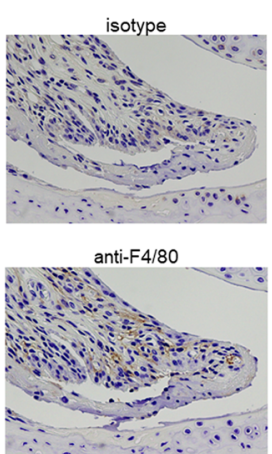

anti-ITIH4

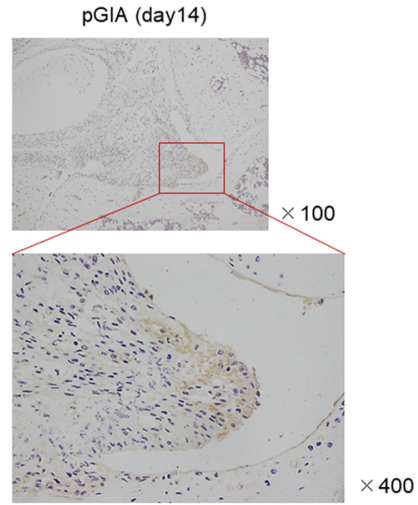

b

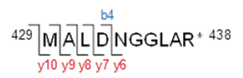

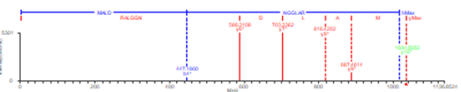
400

\section{e}
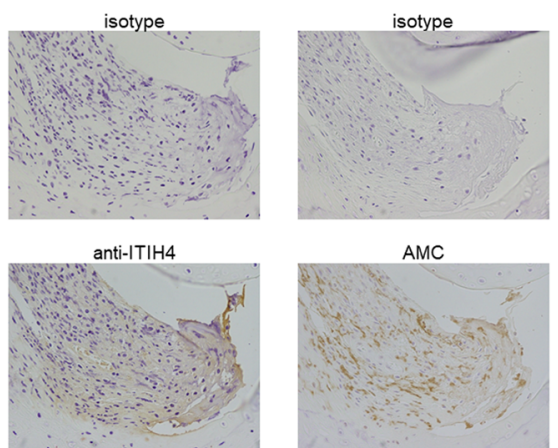

Fig. 2 Identification of citrullinated inter-alpha-trypsin inhibitor heavy chain 4 (ITIH4) in sera and increase of ITIH4 in arthritic joints in peptide glucose-6-phosphate isomerase-induced arthritis ( $\mathrm{pGIA}$ ). a Serum samples from pGIA and control mice at day 14 separated by two-dimensional PAGE, then stained with Coomassie brilliant blue (left) or subjected to Western blot analysis using anti-modified citrulline (AMC) antibodies (right). Citrullinated proteins were detected at approximately $120 \mathrm{kDa}$ and pH $5.3 \mathrm{in} \mathrm{pGIA} \mathrm{but} \mathrm{not} \mathrm{in} \mathrm{controls.} \mathrm{b} \mathrm{The} \mathrm{MS} \mathrm{spectrum} \mathrm{of} \mathrm{ITIH4} 429-438$ and modified peptides bearing the citrullinated arginine (R438) in PGIA. Citrullinated residues were identified by the unmodified b4 ion and the modified y6, y7, y8, y9, and y10 ion confirmed a mass increase of 1.0D. The experiments depicted in (a) and (b) were performed on at least three different samples with similar results. $\mathbf{c}$ Joint sections at day 0 and day 14 were immunohistochemically stained with anti-ITIH4 antibodies in pGIA or control mice. ITIH4 were detected at day 14 in pGIA (indicated by boxed area) but not in naïve or control mice at day 14 . The image on the fourth column is a magnified view of the boxed area in the joint image at day 14 of pGIA. Original magnification $\times 100$ and $\times 400$ as marked. $\mathbf{d}$ Joint sections at day 14 were stained with anti-F4/80 antibodies for detection of macrophages (bottom panel) and isotype control antibodies (top panel) in pGIA. Macrophages colocalized with ITIH4 in some areas in inflamed synovium of PGIA. e Joint sections at day 14 were stained with anti-ITIH4 antibodies (left bottom panel), AMC antibodies (right bottom panel), and respective isotype control antibodies (top panels) in pGIA. ITIH4 colocalized with citrullinated proteins in inflamed synovium of $\mathrm{pGIA}$ in comparative serial sections. Original magnification $\times 400$

detected by anti-F4/80 antibodies in serial sections showed colocalization with ITIH4 in some areas (Fig. 2d), suggesting that synovial macrophages may express ITIH4 in joints of pGIA. Moreover, ITIH4 depositions colocalized with citrullinated proteins in inflamed synovium in comparative serial sections (Fig. 2e), suggesting the presence of citrullinated ITIH4 in joints of pGIA. These results indicate the specific expression of ITIH4 and citrullinated ITIH4 in arthritic joints in pGIA.

\section{Specific increase of citrullinated ITIH4 in sera of patients with RA}

We also investigated the expression of citrullinated proteins in sera of 60 patients with RA and $30 \mathrm{HS}$, as well 
Table 1 Citrullinated inter-alpha-trypsin inhibitor heavy chain 4 was identified in mouse samples by two-dimensional PAGE and nanoUPLC-MS

\begin{tabular}{lllll}
\hline $\begin{array}{l}\text { Protein } \\
\text { ID }\end{array}$ & Protein name & Coverage (\%) & pGIA & MW (kDa) \\
\cline { 3 - 4 } O54882 & Inter-alpha-trypsin inhibitor heavy chain 4 & 55.4 & 63.9 & \\
Position & Peptide sequence & Calculated mass & Observed mass & Arginine \\
429-438 & MALDNGGLA(cit) & 1033.50 & 1033.49 & R438 \\
\hline
\end{tabular}

MW Molecular weight, $p$ GIA Peptide glucose-6-phosphate isomerase-induced arthritis

Calculated mass = calculated mass-to-charge ratio of the modified peptides; observed mass = observed mass-to-charge ratio of the modified peptides

as in 12 patients with osteoarthritis (OA), 15 patients with SLE, and 27 patients with SS (disease controls). As well as in pGIA mice, the $120 \mathrm{kDa}$ band of citrullinated protein was detected in $82 \%$ of patients with RA but in none of the control groups, except for being faintly detected in 2 patients with SS (7\%) and 1 patient with SLE (7\%) (Fig. 3a and Additional file 6: Figure S6a). The levels of this citrullinated protein were significantly higher in patients with RA than in controls (Fig. 3b).

We analyzed the $120 \mathrm{kDa}$ citrullinated protein in patients with RA by 2D-PAGE and MS in the same way as pGIA mice. Using 2D-PAGE and Western blot analysis, we first confirmed the spot of citrullinated protein at $120 \mathrm{kDa}$, similar to pGIA (Fig. 3c). Second, MS of this spot identified ITIH4 with high coverage (Table 2). Analysis of citrulline modifications identified citrullination of several sites in ITIH4 from patients with RA. Citrullinated sites varied among individual samples, but the citrullination of R438 was common among the examined samples $(n=3)$ (Table 2 and Fig. $3 \mathrm{~d})$.

To confirm whether the $120 \mathrm{kDa}$ citrullinated protein was ITIH4, we performed Western blot analysis of serum samples using anti-ITIH4 antibodies. The bands were detected at $120 \mathrm{kDa}$ by anti-ITIH4 antibodies in the same position as the bands detected by AMC antibodies (Fig. 3e). In addition, the levels of total ITIH4 were similar in patients with RA and controls, whereas the levels of citrullinated ITIH4 were specifically increased in patients with RA relative to controls (Fig. 3e). Using ELISA, we also measured total ITIH4 concentrations in sera from 60 patients with RA and $30 \mathrm{HS}$ who were the same individuals used in Western blot analysis. There was no significant difference in total ITIH4 levels between patients with RA and HS (data not shown). These results indicate that the levels of citrullinated ITIH4 rather than total ITIH4 were specifically increased in association with arthritis in sera of patients with RA.

Correlation between citrullinated ITIH4 levels and disease activity in patients with RA

Citrullinated ITIH4 was previously identified as one of various citrullinated proteins in RA synovium [6]; however, its function and association with RA are not clear. We compared the clinical features of patients with RA with or without serum citrullinated ITIH4 as confirmed by Western blot analysis, using a band intensity cutoff value of 0.46 , representing the mean $+3 \mathrm{SD}$ of HS. C-reactive protein (CRP) and rheumatoid factor (RF) levels and Disease Activity Score in 28 joints measured by CRP (DAS28-CRP) scores were significantly higher in citrullinated ITIH4-positive than in ITIH4negative patients $(p=0.047, p=0.011$, and $p=0.040$, respectively), whereas there was no association between citrullinated ITIH4 and anti-CCP antibody titer or antiCCP antibody-positive rate (Table 3 ). These results suggest that high levels of citrullinated ITIH4 were associated with inflammatory markers and disease activity of patients with RA.

In addition, we analyzed the relationship between serum citrullinated ITIH4 and RF/ACPA levels in patients with RA. The levels of citrullinated ITIH4 were significantly higher in $\mathrm{RF}^{+} \mathrm{ACPA}^{+}$patients compared with $\mathrm{RF}^{-} \mathrm{ACPA}^{+}, \mathrm{RF}^{-} \mathrm{ACPA}^{-}$patients ( $\mathrm{p}=0.011, p=0$. 025 , respectively) (Table 4 ), suggesting that citrullinated ITIH4 levels were associated with RF levels in patients with RA. Interestingly, citrullinated ITIH4 were detected in $88 \%$ of $\mathrm{RF}^{-} \mathrm{ACPA}^{-}$patients, although those levels were low (Table 4). It was suggested that positivity of citrullinated ITIH4 could be a useful marker in seronegative patients with RA as well.

To further clarify whether serum citrullinated ITIH4 levels were correlated with the disease activity of patients with RA, we recruited patients who continued biologic treatment for 24 weeks (infliximab, $n=9$; abatacept, $n=8$ ) among citrullinated ITIH4-positive patients. Because treatment with tocilizumab downregulates inflammatory markers sometimes irrespective of arthritis activity, we chose infliximab and abatacept in this experiment. At baseline, the levels of citrullinated ITIH4 were positively correlated with DAS28-CRP, DAS28ESR, Simplified Disease Activity Index, and Clinical Disease Activity Index (Fig. $4 \mathrm{a}-\mathrm{d}$ ). In our comparison of the differences from baseline to 24 weeks after treatment, we found that citrullinated ITIH4 levels were decreased after treatment in responders (Fig. 4e). The difference of 

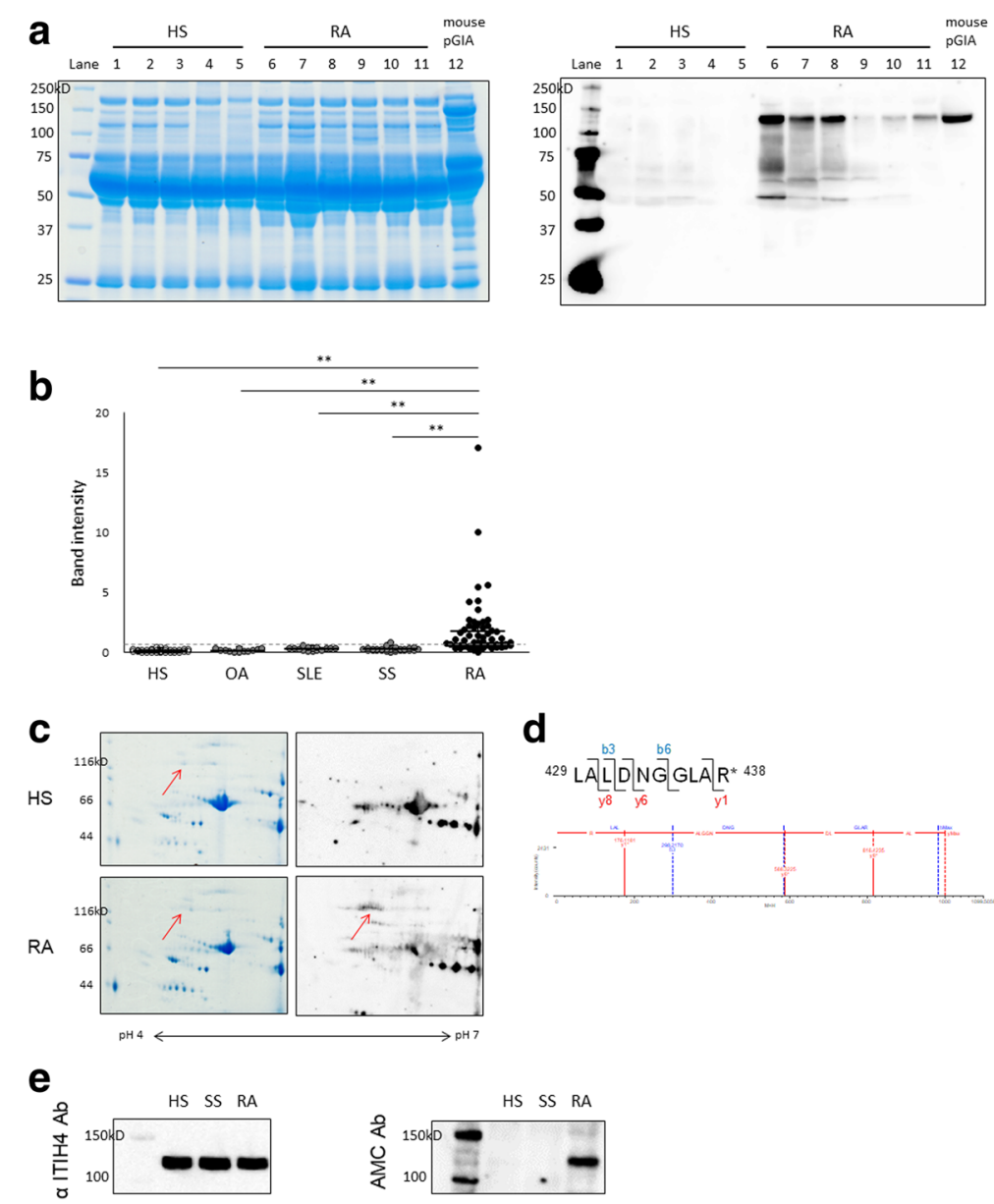

d

Fig. 3 Specific increase of citrullinated inter-alpha-trypsin inhibitor heavy chain 4 (ITIH4) in sera in patients with rheumatoid arthritis (RA). a Sera were separated by SDS-PAGE from patients with RA, healthy subjects (HS), and peptide glucose-6-phosphate isomerase-induced arthritis (pGIA) mice, then stained with Coomassie brilliant blue (CBB) (left) or subjected to Western blot analysis using anti-modified citrulline (AMC) antibodies (right). Citrullinated proteins were detected as $\sim 120 \mathrm{kDa}$ bands in patients with RA and pGIA mice but in none of the HS. CBB staining is shown to demonstrate loading. Lanes 1-5: HS; lanes 6-11: RA; lane 12: pGIA mice at day 14. b The band intensity of $120 \mathrm{kDa}$ citrullinated protein. Sera from patients with RA $(n=60)$, HS $(n=30)$, patients with osteoarthritis (OA) ( $n=12)$, patients with systemic lupus erythematosus (SLE) $(n=15)$, and patients with Sjögren's syndrome (SS) ( $n=27)$ subjected to Western blot analysis using AMC antibodies. Citrullinated proteins at $120 \mathrm{kDa}$ were specifically increased in patients with RA. Each symbol represents a single sample. Horizontal bars represent the mean values; vertical bars represent the SEM; and broken line represents the cutoff value. ${ }^{* *} p<0.01$, N.S. Not significant. c Serum samples from patients with RA and HS separated by two-dimensional PAGE, then stained with CBB (left) or subjected to Western blot analysis using AMC antibodies (right). Citrullinated proteins were detected at $\sim 120 \mathrm{kDa}$ and pH 5.3 in patients with RA but not in HS. d The MS spectrum of $\mathrm{TIH}_{429-438}$ and modified peptides bearing the citrullinated arginine (R438) in patients with RA. Citrullinated residues identified by the unmodified b3 and b6 ions and the modified y1, y6, and y8 ions confirmed a mass increase of 1.0 Da. The experiments shown in (c) and (d) were performed on at least three different samples with similar results. e Serum samples subjected to Western blot analysis using anti-TTIH4 antibodies (Ab) (left) and AMC antibodies (right). The bands were detected at $120 \mathrm{kDa}$ by anti-ITIH4 antibodies as well as AMC antibodies

citrullinated ITIH4 levels was significantly correlated with the difference of DAS28-CRP and DAS28-ESR before and after treatment (Fig. 4f, g). These results suggest that serum citrullinated ITIH4 levels were positively correlated with disease severity and that citrullinated ITIH4 could be a serum marker reflecting disease activity in patients with RA.

\section{Autoantigenicity of citrullinated ITIH4}

Next, we investigated the autoantigenicity of citrullinated ITIH4. We synthesized a citrulline-containing
ITIH $4_{428-447}$ peptide (pITIH4) and the corresponding arginine-containing PITIH4 because R438 was identified as a dominant citrullination site. We assessed antibody reactivities against native or citrullinated PITIH4 by ELISA. On one hand, the levels of anticitrullinated pITIH4 antibody in patients with RA were significantly higher than those of HS (Fig. 5a, right). On the other hand, there was no significant difference in the levels of antinative PITIH4 antibody between patients with RA and HS (Fig. 5a, left). When the cutoff value was determined using sera from HS, $21.7 \%$ of RA sera was shown 
Table 2 Citrullinated inter-alpha-trypsin inhibitor heavy chain 4 was identified in human samples by two-dimensional PAGE and nanoUPLC-MS

\begin{tabular}{lllll}
\hline Protein ID & Protein name & Coverage $(\%)$ & RA & MW (kDa) \\
\cline { 3 - 4 } & & HS & - & \\
\hline B7Z545 & Inter-alpha-trypsin inhibitor heavy chain 4 & 40.0 & 42.3 & \\
B2RMS9 & $\begin{array}{l}\text { Inter-alpha-trypsin inhibitor heavy chain 4 } \\
\text { (inter-alpha globulin inhibitor H4) }\end{array}$ & - & & \\
& & & Observed mass \\
Position & Peptide sequence & Calculated mass & 999.53 & Arginine \\
429-438 & LALDNGGLA(cit) & 999.55 & R438 \\
\hline
\end{tabular}

Abbreviations: HS Healthy subjects, MW Molecular weight, RA Rheumatoid arthritis

Calculated mass = calculated mass-to-charge ratio of the modified peptides; observed mass = observed mass-to-charge ratio of the modified peptides

to be reactive with citrullinated pITIH4. The sensitivity and specificity were $21.7 \%$ and $96.7 \%$, respectively. These results suggest the possibility of an autoimmune reaction to citrullinated ITIH4 in patients with RA.

\section{Discussion}

To our knowledge, this is the first report on high levels of citrullinated ITIH4 in arthritis. ITIH4 is a heavy-chain protein, and ITIHs form inter-alpha-trypsin inhibitors (ITIs) by binding to a common light chain [22, 23]. Members of the ITI family are recruited to extravascular sites, where ITIHs are converted to hyaluronan (HA), to form serum-derived HA-associated protein (SHAP)-HA complexes in HA-rich tissues such as synovial fluid [22, 23]. Researchers in previous studies reported high levels of SHAP-HA complexes in RA serum and synovial fluid, suggesting their association with inflammation through the regulation of adhesion of infiltrating leukocytes [24]. In particular, ITIH4 was identified as one of various citrullinated proteins in RA synovium [6]. In addition, human ITIHs are reported to inhibit the complement system, suggesting a link to the immunocomplex cascades [25]. More recently, high serum levels of anticitrullinated ITIH3 antibodies were found in patients with RA [26].

In the present study, we show that citrullinated ITIH4 was recognized with high specificity in patients with RA as compared with patients with other autoimmune and arthritic diseases or in HS, indicating a potential role for citrullinated ITIH4 in RA pathogenesis. Additionally, in the investigation of autoantigenicity of citrullinated ITIH4, higher levels of anticitrullinated pITIH4 antibody were observed in patients with RA than in HS, whereas there was no significant difference in the levels of antinative pITIH4 antibody. These results suggest that citrullinated ITIH4 may be one of the candidate targets of ACPA and may be involved in the inflammatory response by forming immune complexes, which play an important role in the pathophysiology of RA [27]. ITIH4 has been

Table 3 Comparison of clinical features of patients with rheumatoid arthritis with or without serum citrullinated inter-alpha-trypsin inhibitor heavy chain 4

\begin{tabular}{|c|c|c|c|c|}
\hline & \multirow[t]{2}{*}{ Total } & \multicolumn{2}{|c|}{ Citrullinated ITIH4 } & \multirow[t]{2}{*}{$p$ Value } \\
\hline & & + & - & \\
\hline No. of patients (\%) & 60 & 49 (82\%) & $11(18 \%)$ & \\
\hline Age, years & $52.2 \pm 1.9$ & $51.3 \pm 2.2$ & $55.9 \pm 3.9$ & 0.355 \\
\hline Females, $n(\%)$ & $48(80 \%)$ & $38(78 \%)$ & 10 (91\%) & 0.317 \\
\hline DAS28-CRP score & $3.9 \pm 0.2$ & $4.1 \pm 0.2$ & $3.3 \pm 0.4$ & 0.040 \\
\hline $\mathrm{CRP}, \mathrm{mg} / \mathrm{dl}$ & $2.18 \pm 0.30$ & $2.46 \pm 0.34$ & $0.93 \pm 0.56$ & 0.047 \\
\hline Anti-CCP antibodies, U/ml & $131.6 \pm 21.6$ & $138.9 \pm 24.6$ & $93.4 \pm 39.0$ & 0.462 \\
\hline Positive, $n(\%)$ & $46(82 \%)$ & $39(83 \%)$ & $7(78 \%)$ & 0.709 \\
\hline $\mathrm{RF}, \mathrm{U} / \mathrm{ml}$ & $210.6 \pm 46.1$ & $242.3 \pm 55.0$ & $69.5 \pm 35.5$ & 0.011 \\
\hline MMP-3, ng/ml & $198.5 \pm 21.8$ & $214.7 \pm 25.7$ & $127.9 \pm 20.1$ & 0.122 \\
\hline PSL use, $n$ (\%, mean dose in $\mathrm{mg} /$ day $)$ & $51(85 \%, 6.9)$ & $41(84 \%, 7.1)$ & $10(91 \%, 6.4)$ & 0.544 \\
\hline MTX use, $n$ (\%, mean dose in mg/week) & $40(67 \%, 10.1)$ & $31(63 \%, 10.1)$ & $10(91 \%, 10.0)$ & 0.075 \\
\hline
\end{tabular}

Abbreviations: ITIH4 Inter-alpha-trypsin inhibitor heavy chain 4, DAS28-CRP Disease Activity Score in 28 joints as measured by C-reactive protein, CRP C-reactive protein, CCP Cyclic citrullinated peptide, RF Rheumatoid factor, MMP-3 Matrix metalloproteinase 3, PSL Prednisolone, MTX Methotrexate

Values are mean \pm SEM. The cutoff value for positive citrullinated ITIH4 was band intensity of 0.46 , representing the mean +3 SD of healthy subjects 
Table 4 Relationship between serum citrullinated inter-alpha-trypsin inhibitor heavy chain 4 and rheumatoid factor/anticitrullinated protein antibodies levels in patients with rheumatoid arthritis

\begin{tabular}{lllll}
\hline & $\mathrm{RF}^{+} \mathrm{ACPA}^{+}$ & $\mathrm{RF}^{+} \mathrm{ACPA}$ & $\mathrm{RF}^{-} \mathrm{ACPA}^{+}$ & $\mathrm{RF}^{-} \mathrm{ACPA}^{-}$ \\
\hline No. of patients (\%) & $42(75 \%)$ & $2(4 \%)$ & $4(7 \%)$ & $8(14 \%)$ \\
Cit-ITIH4 level & $2.2 \pm 0.5$ & $1.3 \pm 0.9$ & $0.6 \pm 0.3$ & $0.9 \pm 0.3$ \\
Cit-ITIH4-positive, $n(\%)$ & $38(91 \%)$ & $1(50 \%)$ & $1(25 \%)$ & $7(88 \%)$
\end{tabular}

Abbreviations: Cit-ITIH4 Citrullinated inter-alpha-trypsin inhibitor heavy chain 4, RF Rheumatoid factor, ACPA Anticitrullinated protein antibody Cit-ITIH4 levels are mean \pm SEM of band intensity

reported to have an anti-inflammatory role, such as inhibition of the phagocytosis of polymorphonuclear cells [28]. Regarding the pathogenetic role of citrullinated ITIH4 in RA, another possibility is that citrullination of ITIH4 might suppress the anti-inflammatory effect of ITIH4 in situ, because the biologic activity of many proteins will be affected by their citrullination. Further studies are needed to evaluate the precise role of citrullinated ITIH4 in RA pathogenesis.

Which cells express ITIH4, and how and where is ITIH4 citrullinated? In this study, ITIH4 and macrophages colocalized in some areas in inflamed synovium of pGIA. These results suggest that synovial macrophages may express ITIH4 in pGIA, but there is a limitation of comparing serial sections by IHC. In addition, ITIH4 was also found in synovial or cartilage surface layers in pGIA, suggesting the presence of ITIH4 in the synovial fluid. It is possible that circulating ITIH4 was recruited into synovial fluid, because the ITI family was synthesized by hepatocytes and recruited to extravascular sites such as the synovial fluid [22, 23], and ITIH4 and citrullinated ITIH4 were also detected in RA synovial fluid (our preliminary observations), supporting the possibility of this scenario. In previous reports, peptidyl arginine deiminase 2 (PAD2) and PAD4 enzymes were increased in RA synovium or synovial fluid, and they contributed to protein citrullination in arthritic joints $[5,29,30]$. In this study, we show higher levels of Padi4 in the arthritic joints of pGIA, as well as the
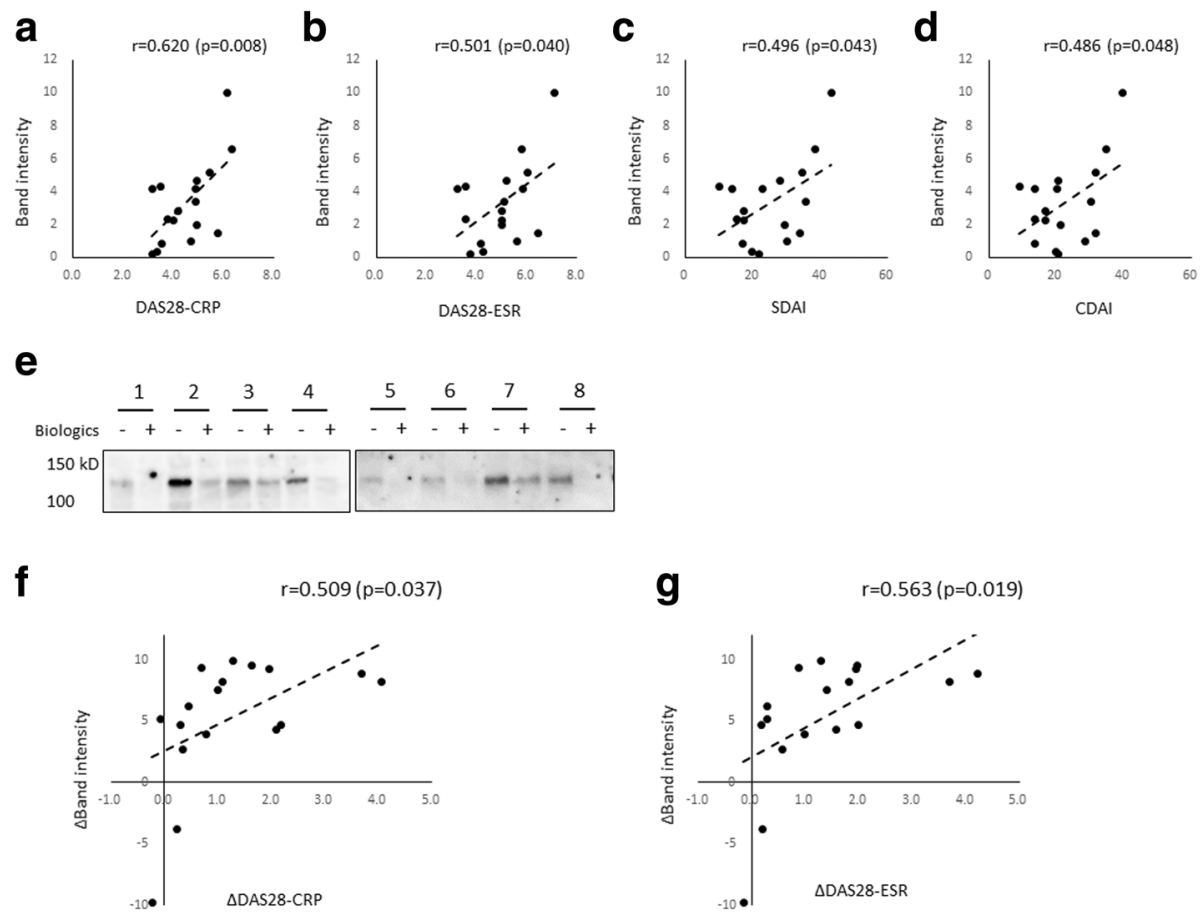

Fig. 4 Relationship between citrullinated inter-alpha-trypsin inhibitor heavy chain 4 (ITIH4) levels and disease activity in patients with rheumatoid arthritis (RA). a-d Correlation between citrullinated ITIH4 levels and disease activity score in 28 joints (DAS28) as measured by C-reactive protein (DAS28-CRP) (a), DAS28-ESR (b), Simplified Disease Activity Index (SDAI) (c), and Clinical Disease Activity Index (CDAI) (d) at baseline in patients with RA ( $n=17)$. e Sera from patients with RA at baseline or 24 weeks after biologic treatment subjected to Western blot analysis using anti-modified citrulline antibodies. Citrullinated ITIH4 levels were decreased after effective treatment. Lanes 1-8: Each individual, lanes 1and 2: abatacept treatment samples, lanes 3-8: infliximab treatment samples, Lanes marked (-): baseline samples; lanes marked (+):posttreatment samples. $\mathbf{f}$ and $\mathbf{g}$ Correlation between the difference of citrullinated ITHH4 levels (expressed as the normalized difference of band intensity) and the difference of (f) DAS28-CRP or (g) DAS28-ESR before and after treatment $(n=17)$. $r$ is the correlation coefficient 


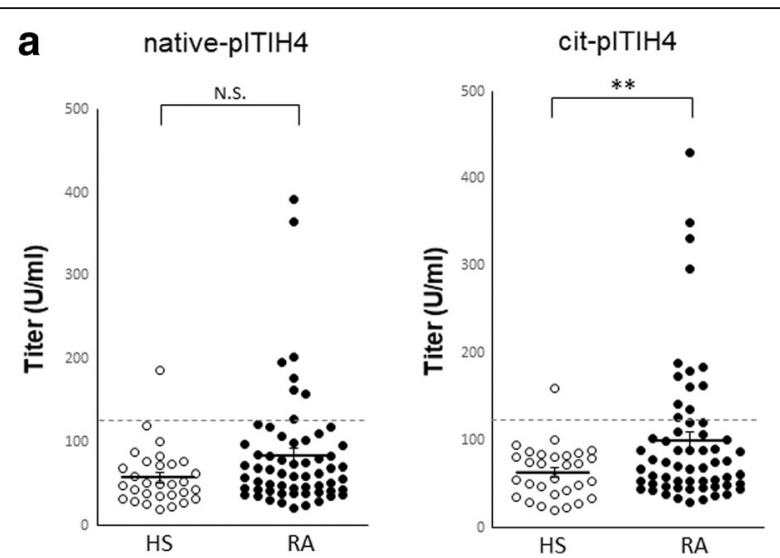

Fig. 5 Autoantigenicity of citrullinated peptide 428-447 inter-alphatrypsin inhibitor heavy chain 4 (pITIH4) in patients with rheumatoid arthritis (RA). a Antibody reactivities against native or citrullinated pITIH4 were analyzed in patients with RA $(n=60)$ and healthy subjects $(H S)(n=30)$ by enzyme-linked immunosorbent assay. Each symbol represents a single sample. Bars show the mean \pm SEM; broken line represents the cutoff value. ${ }^{* *} p<0.01$. N.S. Not significant

expression of ITIH4 and citrullinated ITIH4 in inflamed synovium of pGIA. Taking these findings together, we speculate that citrullination of ITIH4 was induced by aberrant PAD activity in arthritic joints and leaked into the blood circulation.

In this study, on one hand, serum citrullinated ITIH4 levels varied in relation to the clinical findings of pGIA, increasing at day 14 then falling at day 28 . On the other hand, ACPA titers were significantly higher after day 21 , despite the gradual resolution of arthritis. Furthermore, our preliminary studies showed that treatment with $\mathrm{Cl}$-amidine (PAD inhibitor) resulted in suppression of arthritis coupled with a significant decrease in serum citrullinated ITIH4 levels, although there was no significant decrease in ACPA titers in pGIA. In patients with RA, the levels of CRP, RF, and DAS28-CRP were significantly higher in patients with RA positive for serum citrullinated ITIH4. In addition, basal serum citrullinated ITIH4 levels were significantly correlated with disease activity markers in patients with active RA and decreased after effective treatment in correlation with the disease activity. These results suggest that citrullinated ITIH4, not ACPA, seems to fluctuate in parallel with the severity of arthritis and could be a disease-specific biomarker representing the disease activity of patients with RA.

We observed that there were several citrullinated sites in ITIH4, and those sites were not identical between pGIA and patients with RA, and they were different between individuals. However, the R438 site was commonly citrullinated in our analyzed samples. Thus, this position is considered as a dominant citrullinated site. We show that the antibody reactivities against ITIH $4_{428-447}$ peptides containing citrullinated R438 were significantly higher in patients with RA than in HS, whereas there was no significant difference in the reactivities against native ITIH $4_{428-447}$ peptides containing arginine. Furthermore, the levels of anticitrullinated pITIH4 antibody tended to be higher than those of native pITIH4 in patients with RA, although there was no significant difference. There is a possibility that this citrullinated epitope in ITIH4 may be one of the targets of the immune system in RA. Nonetheless, the reactivities against the synthesized linear citrullinated peptides do not necessarily completely reflect the reactivities against the corresponding citrullinated proteins in vivo, and there is also a possibility of antibodies cross-reacting. It is necessary to verify in future studies the antibody reactivities against whole citrullinated ITIH4 or circulating citrullinated epitopes at several other sites.

\section{Conclusions}

We show in the present study the specific expression of citrullinated proteins in joints and sera in pGIA. Notably, we demonstrate that the increase of specific citrullinated protein in sera as citrullinated ITIH4 fluctuated with the arthritis score. Also, in patients with RA, citrullinated ITIH4 levels were specifically increased in sera and significantly correlated with disease activity. Therefore, citrullinated ITIH4 could be a novel biomarker to distinguish RA from other rheumatic diseases and for assessing disease activity in patients with RA.

\section{Additional files}

Additional file 1: Figure S1. DBA/1 mice were immunized with $\mathrm{pGPI}$. (a) The clinical score (mean \pm SEM) of $\mathrm{pGIA}(n=11)$. (b) Sera were obtained once per week between days 0 and 28 from pGIA and control mice. The titers of anti-CCP antibodies were analyzed by ELISA $(n=12-18)$. Each symbol represents a single mouse, and the horizontal and vertical bars represent the mean and SEM values, respectively. ${ }^{*} p<0.05,{ }^{* *} p<0.01$. (TIFF $120 \mathrm{~kb}$ )

Additional file 2: Figure S2. (a) Padi4 gene expression levels in articular tissue samples from pGIA and control mice, analyzed by $\mathrm{PPCR}(n=6)$. Data are mean \pm SEM. (TIFF $97 \mathrm{~kb}$ )

Additional file 3: Figure S3. (a) Serum samples obtained at day 14 from pGIA mice. Western blot analysis was performed without modification of citrulline residues, but no bands of citrullinated proteins were detected (left). Western blot analysis performed with modification of citrulline residues allowed detection of the bands (right). G1-G3 Samples of different mice. (b) Serum of mice immunized with pGPI or CFA control were subjected to the color development reagent assay to measure citrulline content $(n=6)$. Each symbol represents a single mouse. The horizontal and vertical bars represent the mean and SEM values for the group, respectively. (TIFF $149 \mathrm{~kb}$ )

Additional file 4: Figure S4. (a) Sera of pGIA and control mice obtained at day 14 were separated by SDS-PAGE and stained with Coomassie brilliant blue (left) or subjected to Western blot analysis using AMC antibodies (right). Citrullinated proteins were detected at $\sim 120 \mathrm{kDa}$ 
in PGIA mice but not in the control mice. G1, 2: pGIA; C1, 2: control mice (b) The MS spectrum of $\mathrm{ITIH} 4_{429-438}$ and modified peptides bearing the citrullinated arginine (R438) in pGIA. Citrullinated residues were identified by the modified $\mathrm{y}_{6}, \mathrm{y} 7$, and $\mathrm{y} 8$ ion confirmed a mass increase of 1.0 Da. (TIFF $217 \mathrm{~kb}$ )

Additional file 5: Figure S5. (a) Liver tissue sections from naive mice were immunohistochemically stained with anti-ITIH4 antibodies as a positive control to detect ITIH4. (TIFF $263 \mathrm{~kb}$ )

Additional file 6: Figure S6. (a) Sera from patients with RA, HS, patients with OA, patients with SLE, and patients with SS was subjected to Western blot analysis using AMC antibodies. Citrullinated proteins were specifically detected as an $\sim 120 \mathrm{kDa}$ band in patients with RA. (TIFF $238 \mathrm{~kb}$ )

\section{Abbreviations}

2D-PAGE: Two-dimensional PAGE; ABT: Abatacept; ACPA: Anticitrullinated protein antibodies; ACR: American College of Rheumatology; AMC antibodies: Anti-modified citrulline antibodies; BSA: Bovine serum albumin; CBB: Coomassie brilliant blue; CCP: Cyclic citrullinated peptide; CFA: Complete Freund's adjuvant; COLDER: Color development reagent; CRP: C-reactive protein; DAB: 3,3'-Diaminobenzidine; DAS28: Disease Activity Score in 28 joints; ELISA: Enzyme-linked immunosorbent assay; ESR: Erythrocyte sedimentation rate; GAPDH: Glyceraldehyde 3-phosphate dehydrogenase; GIA: Glucose-6-phosphate isomerase-induced arthritis; GPI: Glucose-6-phosphate isomerase; HA: Hyaluronan; HRP: Horseradish peroxidase; HS: Healthy subjects; IFX: Infliximab; IgG: Immunoglobulin G; ITI: Inter-alpha-trypsin inhibitor; ITIH: Inter-alpha-trypsin inhibitor heavy chain; m/z: Mass-to-charge ratio; MMP-3: Matrix metalloproteinase 3;

MTX: Methotrexate; MW: Molecular weight; OA: Osteoarthritis; OD: Optical density; PAD: Peptidyl arginine deiminase; pGIA: Peptide glucose-6phosphate isomerase-induced arthritis; pGPI: Peptide 325-339 glucose-6phosphate isomerase; PSL: Prednisolone; RA: Rheumatoid arthritis; RF: Rheumatoid factor; SDAI: Simplified Disease Activity Index; SHAP: Serumderived hyaluronan-associated protein; SLE: Systemic lupus erythematosus: SS: Sjögren's syndrome; TBST: Tween 20 in Tris-buffered saline; TMB: 3,3',5,5'Tetramethylbenzidine; UPLC: Ultrahigh-performance LC

\section{Acknowledgements}

We thank Dr. F. G. Issa for critical reading of the manuscript.

\section{Funding}

This work was supported by a Grant-in-Aid for Scientific Research (grant number 15 K09518) from the Ministry of Education, Culture, Sports, Science and Technology and by the Japan Society for the Promotion of Science.

\section{Availability of data and materials}

The datasets used and/or analyzed during the present study are available from the corresponding author on reasonable request.

\section{Authors' contribution}

$H K, I M$ and TS designed the study. HK, AO, IK, HE, YT, Aln, and NU performed experiments and collected the data. YKo supervised tissue analysis. HT oversaw patient recruitment. YS and YKu performed LC-MS analysis. Als provided AMC antibodies. HK, IM and TS analyzed the data, and wrote the manuscript. All authors have read and approved the manuscript for publication.

\section{Ethics approval}

Approval for this study was obtained from the local ethics committees of the University of Tsukuba Hospital (reference number H29-041). A signed informed consent to participate was obtained from each participant. All animal experimental protocols were approved by the Institutional Animal Care and Use Committee of the University of Tsukuba (reference number 17-269), and all animal experiments were conducted in accordance with institutional ethics guidelines.

\section{Consent for publication}

A signed informed consent for publication was obtained from each participant.

\section{Competing interests}

The authors declare that they have no competing interests.

\section{Publisher's Note}

Springer Nature remains neutral with regard to jurisdictional claims in published maps and institutional affiliations.

\section{Author details}

${ }^{1}$ Department of Internal Medicine, Faculty of Medicine, University of Tsukuba, 1-1-1 Tennodai, Tsukuba 305-8575, Japan. Environmental Biology Laboratory, Faculty of Medicine, University of Tsukuba, Tsukuba, Japan. ${ }^{3}$ Molecular Regulation of Aging, Tokyo Metropolitan Institute of Gerontology, Tokyo, Japan.

Received: 2 December 2017 Accepted: 7 March 2018

Published online: 10 April 2018

References

1. Firestein GS. Evolving concepts of rheumatoid arthritis. Nature. 2003;423: 356-61

2. Sakkas L, Bogdanos D, Katsiari C. Anti-citrullinated peptides as autoantigens in rheumatoid arthritis—relevance to treatment. Autoimmun Rev. 2014;13: 1114-20.

3. Kuhn KA, Kulik L, Tomooka B, Braschler KJ, Arend WP, Robinson WH, Holers VM. Antibodies against citrullinated proteins enhance tissue injury in experimental autoimmune arthritis. J Clin Invest. 2006:116:961-73.

4. Umeda N, Matsumoto I, Ito I, Kawasaki A, Tanaka Y, Inoue A, Tsuboi H, Suzuki T, Hayashi T, Ito S, Tsuchiya N, Sumida T. Anti-citrullinated glucose-6phosphate isomerase peptide antibodies in patients with rheumatoid arthritis are associated with HLA-DRB1 shared epitope alleles and disease activity. Clin Exp Immunol. 2013;172:44-53.

5. Kinloch A, Lundberg K, Wait R, Wegner N, Lim NH, Zendman AJW, Saxne T, Malmström V Venables PJ. Synovial fluid is a site of citrullination of autoantigens in inflammatory arthritis. Arthritis Rheum. 2008;58:2287-95.

6. van Beers JJBC, Schwarte CM, Stammen-Vogelzangs J, Oosterink E, Božič B, Pruijn GJM. The rheumatoid arthritis synovial fluid citrullinome reveals novel citrullinated epitopes in apolipoprotein E, myeloid nuclear differentiation antigen, and $\beta$-actin. Arthritis Rheum. 2013;65:69-80.

7. James EA, Rieck M, Pieper J, Gebe JA, Yue BB, Tatum M, Peda M, Sandin C, Klareskog L, Malmström V, Buckner JH. Citrulline-specific Th1 cells are increased in rheumatoid arthritis and their frequency is influenced by disease duration and therapy. Arthritis Rheumatol. 2014;66:1712-22.

8. Hill JA, Bell DA, Brintnell W, Yue D, Wehrli B, Jevnikar AM, Lee DM, Hueber W, Robinson WH, Cairns E. Arthritis induced by posttranslationally modified (citrullinated) fibrinogen in DR4-IE transgenic mice. J Exp Med. 2008;205: 967-79.

9. Matsumoto I, Staub A, Benoist C, Mathis D. Arthritis provoked by linked T and B cell recognition of a glycolytic enzyme. Science. 1999:286:1732-5.

10. Schubert D, Maier B, Morawietz L, Krenn V, Kamradt T. Immunization with glucose-6-phosphate isomerase induces T cell-dependent peripheral polyarthritis in genetically unaltered mice. J Immunol. 2004;172:4503-9.

11. Iwanami K, Matsumoto I, Tanaka Y, Inoue A, Goto D, Ito S, Tsutsumi A, Sumida T. Arthritogenic T cell epitope in glucose-6-phosphate isomeraseinduced arthritis. Arthritis Res Ther. 2008;10:R130

12. Iwanami K, Matsumoto I, Tanaka-Watanabe $Y$, Inoue A, Mihara M, Ohsugi $Y$, Mamura M, Goto D, Ito S, Tsutsumi A, Kishimoto T, Sumida T. Crucial role of the interleukin-6/interleukin-17 cytokine axis in the induction of arthritis by glucose-6-phosphate isomerase. Arthritis Rheum. 2008;58:754-63.

13. Matsumoto I, Zhang H, Yasukochi T, Iwanami K, Tanaka Y, Inoue A, Goto D, Ito S, Tsutsumi A, Sumida T. Therapeutic effects of antibodies to tumor necrosis factor-alpha, interleukin-6 and cytotoxic T-lymphocyte antigen 4 immunoglobulin in mice with glucose-6-phosphate isomerase induced arthritis. Arthritis Res Ther. 2008;10:R66.

14. Arnett FC, Edworthy SM, Bloch DA, McShane DJ, Fries JF, Cooper NS, Healey LA, Kaplan SR, Liang MH, Luthra HS. The American Rheumatism Association 1987 revised criteria for the classification of rheumatoid arthritis. Arthritis Rheum. 1988;31:315-24.

15. Aletaha D, Neogi T, Silman AJ, Funovits J, Felson DT, Bingham CO, Birnbaum NS, Burmester GR, Bykerk VP, Cohen MD, Combe B, Costenbader KH, Dougados M, Emery P, Ferraccioli G, Hazes JMW, Hobbs K, et al. 2010 Rheumatoid arthritis classification criteria: an American College of 
Rheumatology/European League Against Rheumatism collaborative initiative. Arthritis Rheum. 2010;62:2569-81.

16. Fujibayashi T, Sugai S, Miyasaka N, Hayashi Y, Tsubota K. Revised Japanese criteria for Sjögren's syndrome (1999): availability and validity. Mod Rheumatol. 2004;14:425-34.

17. Hochberg MC. Updating the American College of Rheumatology revised criteria for the classification of systemic lupus erythematosus. Arthritis Rheum. 1997:40:1725.

18. Banda NK, Kraus D, Vondracek A, Huynh LH, Bendele A, Holers VM, Arend WP. Mechanisms of effects of complement inhibition in murine collageninduced arthritis. Arthritis Rheum. 2002:46:3065-75.

19. Asaga $\mathrm{H}$, Ishigami A. Protein deimination in the rat brain: generation of citrulline-containing proteins in cerebrum perfused with oxygen-deprived media. Biomed Res. 2000;21:197-205.

20. Knipp M, Vasák M. A colorimetric 96-well microtiter plate assay for the determination of enzymatically formed citrulline. Anal Biochem. 2000;286: 257-64.

21. Umeda N, Matsumoto I, Kawaguchi H, Kurashima Y, Kondo Y, Tsuboi H, Ogishima H, Suzuki T, Kagami Y, Sakyu T, Ishigami A, Maruyama N, Sumida T. Prevalence of soluble peptidylarginine deiminase 4 (PAD4) and anti-PAD4 antibodies in autoimmune diseases. Clin Rheumatol. 2016;35:1181-8.

22. Zhuo L, Hascall VC, Kimata K. Inter-alpha-trypsin inhibitor, a covalent protein-glycosaminoglycan-protein complex. J Biol Chem. 2004;279:3807982.

23. Zhuo L, Kimata K. Structure and function of inter-a-trypsin inhibitor heavy chains. Connect Tissue Res. 2008:49:311-20.

24. Yingsung W, Zhuo L, Morgelin M, Yoneda M, Kida D, Watanabe $H$, Ishiguro $\mathrm{N}$, Iwata H, Kimata K. Molecular heterogeneity of the SHAP-hyaluronan complex. Isolation and characterization of the complex in synovial fluid from patients with rheumatoid arthritis. J Biol Chem. 2003;278:32710-8.

25. Okroj M, Holmquist E, Sjölander J, Corrales L, Saxne T, Wisniewski H-G, Blom AM. Heavy chains of inter alpha inhibitor (lal) inhibit the human complement system at early stages of the cascade. J Biol Chem. 2012;287: 20100-10.

26. Liao CC, Chou PL, Cheng CW, Chang YS, Chi WM, Tsai KL, Chen WJ, Kung TS, Tai CC, Lee KW, Chen YC, Lin CY. Comparative analysis of novel autoantibody isotypes against citrullinated-inter-al pha-trypsin inhibitor heavy chain $3(\mathrm{ITIH} 3)^{542-556}$ peptide in serum from Taiwanese females with rheumatoid arthritis, primary Sjögren's syndrome and secondary Sjögren's syndrome in rheumatoid arthritis. J Proteome. 2016;141:1-11.

27. Sokolove J, Zhao X, Chandra PE, Robinson WH. Immune complexes containing citrullinated fibrinogen costimulate macrophages via Toll-like receptor 4 and Fcy receptor. Arthritis Rheum. 2011;63:53-62.

28. Choi-Miura NH, Takahashi K, Yoda M, Saito K, Hori M, Ozaki H, Mazda T, Tomita M. The novel acute phase protein, IHRP, inhibits actin polymerization and phagocytosis of polymorphonuclear cells. Inflamm Res. 2000;49:305-10.

29. De Rycke L, Nicholas AP, Cantaert T, Kruithof E, Echols JD, Vandekerckhove $B$, Veys EM, De Keyser F, Baeten D. Synovial intracellular citrullinated proteins colocalizing with peptidyl arginine deiminase as pathophysiologically relevant antigenic determinants of rheumatoid arthritis-specific humoral autoimmunity. Arthritis Rheum. 2005;52:2323-30.

30. Foulquier C, Sebbag M, Clavel C, Chapuy-Regaud S, Al Badine R, Méchin MC, Vincent C, Nachat R, Yamada M, Takahara H, Simon M, Guerrin M, Serre G. Peptidyl arginine deiminase type 2 (PAD-2) and PAD-4 but not PAD-1, PAD-3, and PAD-6 are expressed in rheumatoid arthritis synovium in close association with tissue inflammation. Arthritis Rheum. 2007;56:3541-53.

\section{Submit your next manuscript to BioMed Central and we will help you at every step:}

- We accept pre-submission inquiries

- Our selector tool helps you to find the most relevant journal

- We provide round the clock customer support

- Convenient online submission

- Thorough peer review

- Inclusion in PubMed and all major indexing services

- Maximum visibility for your research

Submit your manuscript at www.biomedcentral.com/submit

C Biomed Central 